\title{
SWELLING OF THE CORNEA IN VIVO AFTER THE DESTRUCTION OF ITS LIMITING LAYERS*
}

\author{
BY \\ D. M. MAURICE AND A. A. GIARDINI \\ Ophthalmological Research Unit (Medical Research Council), Institute of \\ Ophthalmology, London, and Clinica Oculistica, Università di Parma, Italy
}

IT is well known that the cornea will swell if excised and placed in an aqueous solution, and the attention of many investigators has been turned to the reason for its unswollen state in the normal eye. It is generally accepted that the explanation lies in the properties of the limiting membranes of the cornea and various theories have been propounded as to the mechanism of their action. Leber (1873) based his explanation on the high degree of impermeability to aqueous humour shown by him to be possessed by the endothelium, and Cogan and Kinsey (1942), finding that water could pass freely across both limiting membranes of the cornea and that they were semipermeable, accounted for the deturgesced state of the normal stroma by the removal of water from it by hypertonic tears and aqueous humour. Davson (1949a), and Maurice (1951) considered that this explanation was improbable and concluded that some active extrusion must occur at the membranes. In the light of this hypothesis it would be interesting to investigate the effect of metabolic poisons on the degree of corneal hydration, as this could be an indication of the processes involved in the removal of water from this tissue.

In order to assess the degree of impairment of the function of the limiting membranes, it was decided to find out as a preliminary step what was the potential swelling that could occur in vivo if this function was completely abolished by their mechanical removal. At the same time techniques for measuring the swelling could be developed which would be available for the subsequent experiments with poisons.

The only quantitative investigations of corneal swelling after removal of the epithelium or endothelium, of which we are aware, are those of Kinsey and Cogan (1942), which were restricted to the excised tissues. As far as removal of these membranes in vivo is concerned, Leber (1873) noticed a considerable increase in thickness after removal of the endothelium. There do not, however, appear to be any corresponding observations for the epithelium in the

* Received for publication July 5, 1951. 
literature, for both Davson (1949b) and Adler (1950) refer in their recent text-books to the common belief that stripping the epithelium is not followed by an appreciable swelling.

\section{METHODS}

Rabbits were used throughout these experiments. To remove the corneal epithelium, the animal was anaesthetized with nembutal, the eye subluxated, and the surface of the cornea scraped with a scalpel. Fluorescein was then instilled and any unstained areas were given further treatment. After the removal was completed, the eyelids were kept closed except during the actual examination of the cornea; when the animal had recovered from the anaesthesia, in the experiments that lasted many days, it was allowed normal use of its eyelids.

To destroy the endothelium, use was made of a hypodermic needle of which the pointed end had been heated and bent to a curvature rather greater than that of the cornea. This was pushed through the cornea near the limbus and its endothelial surface thoroughly rubbed with the outer surface of the bend. A deep anterior chamber was maintained in order to permit free movement of the needle, by connecting it to a source of physiological saline above the normal intra-ocular pressure.

In the first experiments the change in size of the scraped cornea at the end of the experimental time was estimated by excising it, and comparing its weight with that of the control cornea from the same animal. Both corneae were cut round the pigmented circle at the limbus, superficially dried with filter paper and weighed immediately on a torsion balance to the nearest $0.5 \mathrm{mg}$. Agreement between untouched pairs of corneae was excellent, being within $1 \mathrm{mg}$. When it became evident that a very large number of animals would have to be sacrificed in order to determine a complete cycle of corneal behaviour, an optical method (Maurice and Giardini, 1951) was developed to express the changes that occurred in individual eyes in terms of alterations in the thickness of the corneae.

\section{Results}

The average thickness of the cornea in sixteen animals was $0.375 \mathrm{~mm}$. (standard error of the mean $0.01 \mathrm{~mm}$.) assuming an average refractive index of 1.38. In any animal this was appreciably constant round the greater part of the horizontal meridian. This figure is to be preferred to a lower value $(0.33 \mathrm{~mm}$.) derived from the area, weight, and density of some corneae by Maurice (1951).

Changes after Removal of the Epithelium.-In Fig. 1, Curve A shows the average (median) change in the optical thickness of seventeen corneae from eleven animals in the first few hours after the removal of the epithelium. There is an immediate drop of roughly 8 per cent. corresponding to the thickness of the epithelium. After this there is a period of rapid swelling, which is initially about $\frac{1}{2}$ per cent. of its total thickness per minute, but after one hour the rate of increase becomes markedly lower. The swelling curve did not rise steadily in the case of every animal studied; sometimes, for no evident reason, periods of rapid swelling alternated with periods of as long as one hour when the thickness remained constant or increased slowly. The extreme ranges exhibited by the individual curves are indicated in Fig. 1 by arrows. 


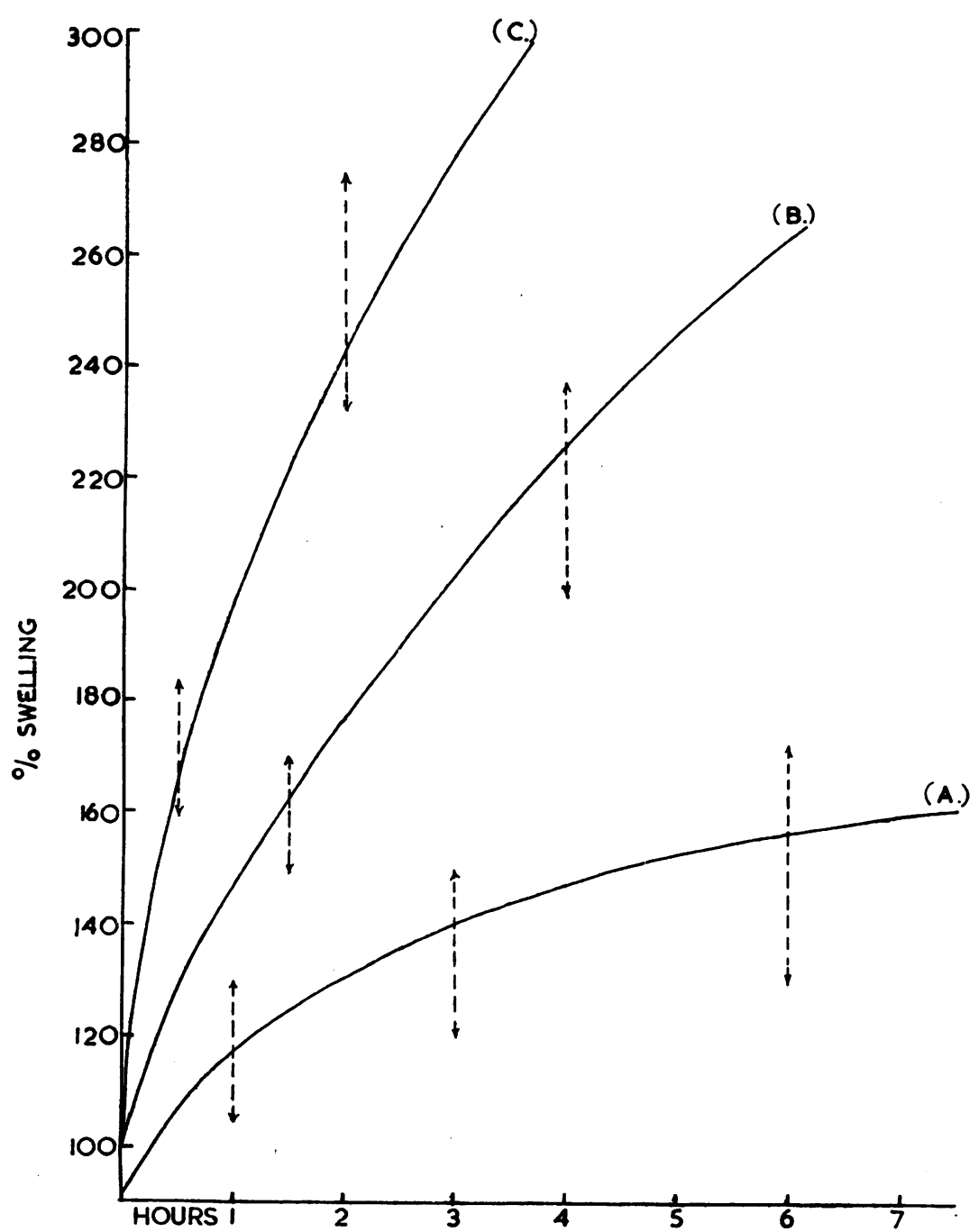

FIG. 1.-Changes in thickness of cornea after removal of limiting layers. Median curves are shown, arrows indicating range of experimental curves. Thickness of untouched cornea $=100$.

(A) Epithelium removed (17 eyes)

(C) Endothelium and Descemet's membrane removed (4 eyes)

The relative increases in weight seemed to be generally smaller than increases in optical thickness for experimental times longer than one hour. That this is a real effect was shown by comparative weight and thickness measurements on the same eye in some animals, differences of 50 to 100 per cent. in favour of the optical measurement being recorded. There 
must therefore be an unequal swelling over the cornea, the value as recorded optically in the centre being greater than that at the edges, where it is perhaps restrained by its structural continuity with the sclera.

The variation in optical thickness at the centre of the cornea from the initial removal of the epithelium until complete recovery is shown in a typical instance in Fig. 2. The average of all the curves is not presented, since the recovery phase starts at a different time in each case, so that the shape of the latter part of the curve would be distorted if this treatment were applied.

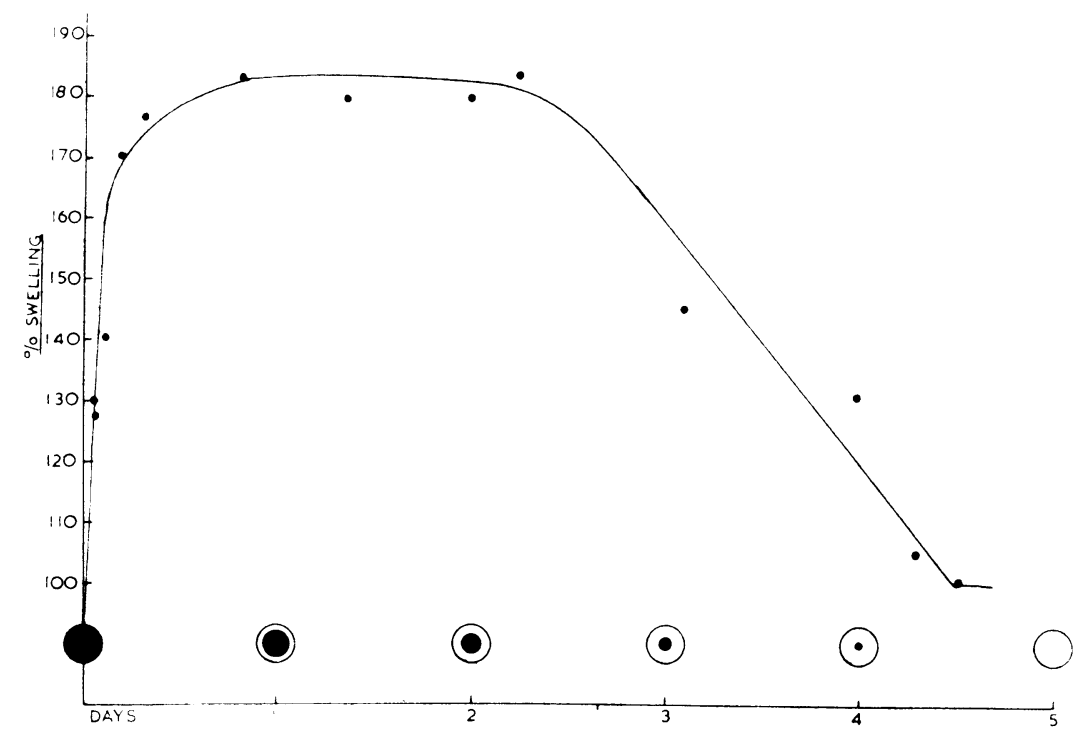

FIG. 2.-Changes in thickness of cornea, at its centre after removal of epithelium, in a typical instance. Points represent experimental determinations. Diagrams below curve show roughly the extent of epithelial regrowth.

In every case studied (thirteen eyes), the swelling curve reached a plateau before the end of 24 hrs; this plateau extended for 2 to 3 days, the recovery phase beginning on the average after $2 \frac{1}{2}$ days. The average maximum swelling was of the order of 200 per cent. of the scraped thickness with a range of 160 to 235 per cent. However, when the swelling rose above 220 per cent. the measurements became increasingly difficult owing to impairment of the endothelial reflex, the result either of endothelial damage, or more probably, to the appreciable cloudiness of the stroma. Generally, it was found that both eyes of the same animal gave similar curves until the end of the plateau was reached.

The greatest variations between eyes occurred during the recovery phase. Some corneae regained their original thickness in one day, others were still more or less swollen up to 4 days after the start of recovery. The long recovery period was not apparently associated with a high degree of swelling, but occurred in cases in which the anterior surface showed 
imperfect regeneration probably on account of mild trauma or slight inflammatory reaction. It is possible also that the steepness of the recovery curve, as measured centrally, was reduced in some cases as a result of an eccentric regrowth of the epithelium. In all cases the cornea ultimately resumed its original thickness; in some cases the epithelium of only one of the eyes was removed, and measurements on the thickness of the opposite cornea showed no significant variations during the experimental period.

Most eyes were stained with fluorescein before a measurement of thickness was taken. Initially, this ensured that the removal of the epithelium was complete, and later it showed the correspondence between the return to normal thickness and the extent of epithelial regrowth. This relationship, as far as it could be estimated visually, is shown by the diagrams under the curve in Fig. 2. At the same time the local variations in thickness in the neighbourhood of the stained area could be seen from the shape of the optical section. In general, the thickness was uniform over this area and underwent an abrupt reduction just beyond its boundary, whence measurements showed a gradual resumption of the normal thickness towards the corneal periphery.

During the first hour or two after the stripping off of the epithelium, there was in every case a fairly strong, clear lacrimation. This is of interest, since in other experiments it was not possible to obtain such an excretion with more usual forms of stimulation, such as tear-gas and lacrimal nerve excitation.

Changes after Removal of the Endothelium.-In this series the swelling after the first few hours was more than the instrument was capable of measuring, and observations were therrefore restricted to this initial period with the animal constantly under anaesthesia. Measurements were carried out where the swelling appeared to be a maximum and were completed successfully in ten eyes.

The results fell into two quite distinct groups of six cases and four cases, and the median curve of each is plotted as the Curves ' $\mathrm{B}$ ' and ' $\mathrm{C}$ ' in Fig. 1. No true endothelial reflex could be seen in the cases in Group C, and the rear edge of the optical section had to be used in the measurement of the thickness. This suggested that the greater rate of swelling was due to the rupture of Descemet's membrane, and in two cases (in one of which the posterior corneal surface was deliberately scratched with the point of the needle) this was confirmed by inspection of sections of the excised cornea under the microscope, which disclosed that this layer was absent over a large area. Comparative observations on four of the eyes which had the lower rate of swelling showed that Descemet's membrane was intact, and confirmed that the endothelial cells had been removed over the greater part of the surface. In contrast to the behaviour after epithelial removal, the increase in thickness appeared to be continuous, except in one or two areas where it was found or suspected that endothelial destruction was not complete.

The anterior chamber was sufficiently reformed to allow measurements to be taken within 5 to $10 \mathrm{~min}$. after the operation, and subsequently there was generally a period of raised ocular tension. That this was not in 
itself responsible for an increased cornear thickness was checked by taking measurements on a peripheral undamaged part of the cornea where it was seen to remain unchanged.

\section{Discussion}

The rate of increase of thickness when the endothelial cells are destroyed (100 per cent. in $3 \mathrm{hrs}$ ) is somewhat below that found for the increase of weight (150 per cent. in $3 \mathrm{hrs}$ ) when excised corneae from which the endothelium had been rubbed, were divided in two and placed in saline at $37^{\circ} \mathrm{C}$.; this figure was not markedly different for solutions of 0.9 and 2.0 per cent. $\mathrm{NaCl}$ at $p \mathrm{H}$ values of 7.4 and 9.0. Such a difference is to be expected in vitro, because of the possibility of the absorption of fluid at the cut periphery. By analogy with these excised pieces, the swelling $24 \mathrm{hrs}$ after the destruction of the endothelium in the living eye would be expected to be five times the original thickness.

On the other hand, the initial rate of swelling after removal of the epithelium is some six times less than that which occurs on removal of the endothelium and Descemet's membrane together, and the ultimate increase is lower by a similar factor, whereas a similarity might be expected, since- the rabbit lacking a Bowman's membranethe stroma is equally exposed in either case.

It is not difficult to find causes which could contribute to the diminished rate of thickening, the most obvious being:

(1) insufficiency of tears,

(2) evaporation,

(3) pressure on the stroma between the eyelids and Descemet's membrane,

(4) relative impermeability of the outer layers of the stroma,

(5) greater difficulty in swelling outwards from the centre of the eye than inwards into the anterior chamber, owing to tension in the fibres,

(6) secretion of fluid out of the cornea by the endothelium.

Further investigations are being made to determine which of these possibilities are of importance.

\section{SUMMARY}

(1) The rate of increase of thickness of the rabbit's cornea after removal of its limiting layers was determined by an optical method.

(2) The average rate of increase was initially about 0.5 per cent. of the thickness per minute for the epithelium, 2 per cent. per minute for the endothelium, and 3 per cent. per minute for the endothelium and Descemet's membrane together.

(3) After the removal of the epithelium the cornea swelled on the average to double its thickness, at which level it remained for about 
2 days, and then regained its original thickness in one or more days simultaneously with the regrowth of the epithelium.

\section{REFERENCES}

Adler, F. H. (1950). " "Physiology of the Eye ", p. 58. Kimpton, London.

Cogan, D. G., and Kinsey, V. E. (1942), Arch. Ophthal., Chicago, 28, 661.

DAvson, H. (1949a). British Journal of Ophthalmology, 33, 175.

(1949b). "Physiology of the Eye ", p. 49. Churchill, London.

Kinsey, V. E., and CogAN, D. G. (1942). Arch. Ophthal., Chicago, 28, 449.

LEBER, T. (1873). v. Graefes Arch. Ophthal., 19, 87.

Maurice, D. M. (1951). J. Physiol., 112, 367.

and GiardinI, A. A. (1951). British Journal of Ophthalmology, 35, 169. 